\title{
A clinicopathological and molecular analysis of 200 traditional serrated adenomas
}

\author{
Mark L Bettington ${ }^{1,2,3}$, Neal I Walker ${ }^{2,3}$, Christophe Rosty ${ }^{2,3,4}$, Ian S Brown ${ }^{3,5}$, \\ Andrew D Clouston $2,3,5$, Diane M McKeone ${ }^{1}$, Sally-Ann Pearson ${ }^{1}$, Kerenaftali Klein ${ }^{6}$, \\ Barbara A Leggett ${ }^{1,2,7}$ and Vicki LJ Whitehall ${ }^{1,2,8}$
}

${ }^{1}$ The Conjoint Gastroenterology Laboratory, QIMR Berghofer Medical Research Institute, Brisbane, QLD, Australia; ${ }^{2}$ The School of Medicine, The University of Queensland, Brisbane, QLD, Australia; ${ }^{3}$ Envoi Specialist Pathologists, Brisbane, QLD, Australia; ${ }^{4}$ Genetic Epidemiology Laboratory, Department of Pathology, The University of Melbourne, Carlton, VIC, Australia; ${ }^{5}$ Department of Anatomical Pathology, Pathology Queensland, Brisbane, QLD, Australia; ${ }^{6}$ Statistics Unit, QIMR Berghofer Medical Research Institute, Brisbane, QLD, Australia; ${ }^{7}$ The Royal Brisbane and Women's Hospital, Brisbane, QLD, Australia and ${ }^{8}$ Department of Chemical Pathology, Pathology Queensland, Brisbane, QLD, Australia

\begin{abstract}
The traditional serrated adenoma is the least common colorectal serrated polyp. The clinicopathological features and molecular drivers of these polyps require further investigation. We have prospectively collected a cohort of $\mathbf{2 0 0}$ ordinary and advanced traditional serrated adenomas and performed BRAF and KRAS mutational profiling, CpG island methylator phenotype analysis, and immunohistochemistry for a panel of 7 antibodies (MLH1, $\beta$-catenin, p53, p16, Ki67, CK7, and CK20) on all cases. The mean age of the patients was 64 years and $50 \%$ were female. Of the polyps, $71 \%$ were distal. Advanced histology (overt dysplasia or carcinoma) was present in $19 \%$ of cases. BRAF mutation was present in $67 \%$ and KRAS mutation in $22 \%$. BRAF mutant traditional serrated adenomas were more frequently proximal (39\% versus $2 \% ; \boldsymbol{P} \leq 0.0001)$, were exclusively associated with a precursor polyp (57\% versus $0 \% ; P \leq 0.0001)$, and were more frequently $\mathrm{CpG}$ island methylator phenotype high $(60 \%$ versus $16 \% ; P \leq 0.0001)$ than KRAS mutant traditional serrated adenomas. Advanced traditional serrated adenomas retained MLH1 expression in $97 \%$, showed strong p53 staining in $55 \%$, and nuclear $\beta$-catenin staining in $40 \%$. P16 staining was lost in the advanced areas of $55 \%$ of $B R A F$ mutant traditional serrated adenomas compared with $10 \%$ of the advanced areas of KRAS mutant or BRAF/KRAS wildtype traditional serrated adenomas. BRAF and KRAS mutant traditional serrated adenomas are morphologically related but biologically disparate polyps with distinctive clinicopathological and molecular features. The overwhelming majority of traditional serrated adenomas retain mismatch repair enzyme function indicating a microsatellite-stable phenotype. Malignant progression occurs via TP53 mutation and Wnt pathway activation regardless of mutation status. However, CDKN2A (encoding the p16 protein) is silenced nearly exclusively in the advanced areas of the BRAF mutant traditional serrated adenomas. Thus, the BRAF mutant traditional serrated adenoma represents an important precursor of the aggressive BRAF mutant, microsatellite-stable subtype of colorectal carcinoma.

Modern Pathology (2015) 28, 414-427; doi:10.1038/modpathol.2014.122; published online 12 September 2014
\end{abstract}

The serrated neoplasia pathway accounts for 15 to $35 \%$ of colorectal carcinoma. ${ }^{1-3}$ Well-established molecular drivers of this pathway are MAP kinase pathway activation, a critical early event resulting

Correspondence: Dr ML Bettington, BSc, MBBS, FRCPA, The Conjoint Gastroenterology Laboratory, Level 7, CBCRC, QIMR Berghofer Medical Research Institute, 300 Herston Road, Herston, Brisbane, QLD 4006, Australia.

E-mail: Mark.Bettington@qimrberghofer.edu.au

Received 1 May 2014; revised 13 June 2014; accepted 15 June 2014; published online 12 September 2014 from either activating $B R A F$ or $K R A S$ mutation, ${ }^{4}$ and the $\mathrm{CpG}$ island methylator phenotype, a coordinate methylation of $\mathrm{CpG}$ islands in the promoter regions of many genes that results in gene silencing. ${ }^{4-6}$ The CpG island methylator phenotype is particularly relevant to carcinogenesis when affecting tumorsuppressor genes. ${ }^{5} \mathrm{MLH} 1$ is the best known of these, with silencing leading to microsatellite instability. This is frequently observed in the malignant transformation of sessile serrated adenomas. However, MLH1 methylation and microsatellite instability are not prerequisites of serrated neoplasia. 
Traditional serrated adenomas remain the least understood of the serrated polyps, probably reflecting their rarity, accounting for $<1 \%$ of colorectal polyps in most series. ${ }^{7-10}$ They were first defined by Longacre and Fenoglio-Preiser ${ }^{7}$ as serrated adenomas, describing polyps with mixed hyperplastic and adenomatous features, a subset of which showed what is now considered the 'typical cytology' of a traditional serrated adenoma, namely cells with abundant eosinophilic cytoplasm and centrally placed pencillate nuclei. ${ }^{7}$ Since then, a diverse range of polyps including sessile serrated adenoma, sessile serrated adenoma with dysplasia, and tubulovillous adenoma with prominent serration have been misclassified as traditional serrated adenomas. ${ }^{11}$ Publications by Torlakovic et al ${ }^{12,13}$ in 2003 and 2008 improved diagnostic reproducibility by characterizing the sessile serrated adenoma and identifying key features of the traditional serrated adenoma, in particular ectopic crypt formations. The fourth edition of the WHO Classification of Tumors of the Digestive Tract emphasizes protuberant and villiform growth patterns and ectopic crypt formations in the diagnosis of the traditional serrated adenoma. ${ }^{11}$ Typical cytology is recognized as a frequent but not requisite feature.

In our experience, polyps with flat growth and absent ectopic crypt formations, but with classical traditional serrated adenoma cytology and slit-like serration, are relatively common but inconsistently classified. Many pathologists consider the typical eosinophilic cell of the traditional serrated adenoma to be inherently dysplastic, yet these cells are not morphologically atypical, do not display mitotic activity, and show absent or minimal proliferative activity by Ki67 staining. ${ }^{11,13}$ Moreover, a subset of traditional serrated adenomas develop discrete areas of morphologically overt dysplasia. ${ }^{14-16}$ How to classify these advanced traditional serrated adenomas and how to separate them from ordinary traditional serrated adenomas in routine practice has been inadequately addressed. The origin of the traditional serrated adenomas is also unclear. Whereas some probably arise de novo, many appear to arise in a precursor polyp, especially microvesicular hyperplastic polyps or sessile serrated adenomas. ${ }^{13,16-18}$

The traditional serrated adenoma also shows more molecular heterogeneity than most other polyps. The frequency of BRAF and KRAS mutation and CpG island methylator phenotype high versus CpG island methylator phenotype low or negative is variable in the literature, unusual for a polyp that, above issues aside, is morphologically fairly uniform. ${ }^{14,16,17,19,20}$ The reasons for, and the significance of, this heterogeneity have not been investigated. Finally, the pathways by which traditional serrated adenomas progress to carcinoma have not been extensively studied.

Herein, we provide a detailed clinicopathological, morphological, and molecular examination of a series of 200 traditional serrated adenomas. We aimed to address the above morphological issues and to interrogate the molecular features of these polyps, with a two-part focus on (1) BRAF versus $K R A S$ mutant traditional serrated adenomas and (2) ordinary versus advanced traditional serrated adenomas. We find that traditional serrated adenomas can be flat and frequently arise in a precursor microvesicular hyperplastic polyp or sessile serrated adenoma. Furthermore, although BRAF and KRAS mutant traditional serrated adenomas are morphologically related, they are biologically distinct polyps, with differing clinicopathological and molecular features that culminate in different subtypes of colorectal carcinoma.

\section{Materials and methods}

\section{Patients and Samples}

A total of 200 traditional serrated adenomas from 196 patients were included in the study. Cases were prospectively collected between June 2007 and June 2013 during routine reporting by one author (NW) at Envoi Specialist Pathologists. All cases were reviewed by two pathologists (MB and NW) and included only if both pathologists were in agreement on the diagnosis. The series included traditional serrated adenomas removed by polypectomy, endoscopic mucosal resection, transanal endoscopic microsurgery, and colectomy. Cases from patients with known inflammatory bowel disease or a polyposis syndrome were excluded. Clinicopathological data including patient age, gender, polyp size, and location were collected from a combination of the pathology request form, specimen container, and pathology report. In this study, proximal includes cecum, ascending colon, hepatic flexure, and transverse colon; distal includes splenic flexure, descending colon, sigmoid colon, and rectum. As a control group, 50 tubulovillous adenomas without morphological evidence of serration were collected. Tubulovillous adenomas were selected as the control group as they more closely simulate the morphology of the traditional serrated adenoma than do tubular adenomas. The study was approved by the ethics committee of QIMR Berghofer Medical Research Institute (P1298).

\section{Histopathological Inclusion Criteria}

Inclusion criteria were based on previously published features. . $^{3,11-13}$ All polyps displayed at least two of the following three features: (1) typical cytology, (2) slit-like epithelial serrations, and (3) ectopic crypt formations, with at least one feature evident in $>50 \%$ of the polyps (Figures $1 \mathrm{a}$ and $\mathrm{b}$ ).

Typical cytology referred to cells with abundant brightly eosinophilic cytoplasm with centrally placed, pencillate nuclei ${ }^{7}$ (Figure 1b); slit-like 

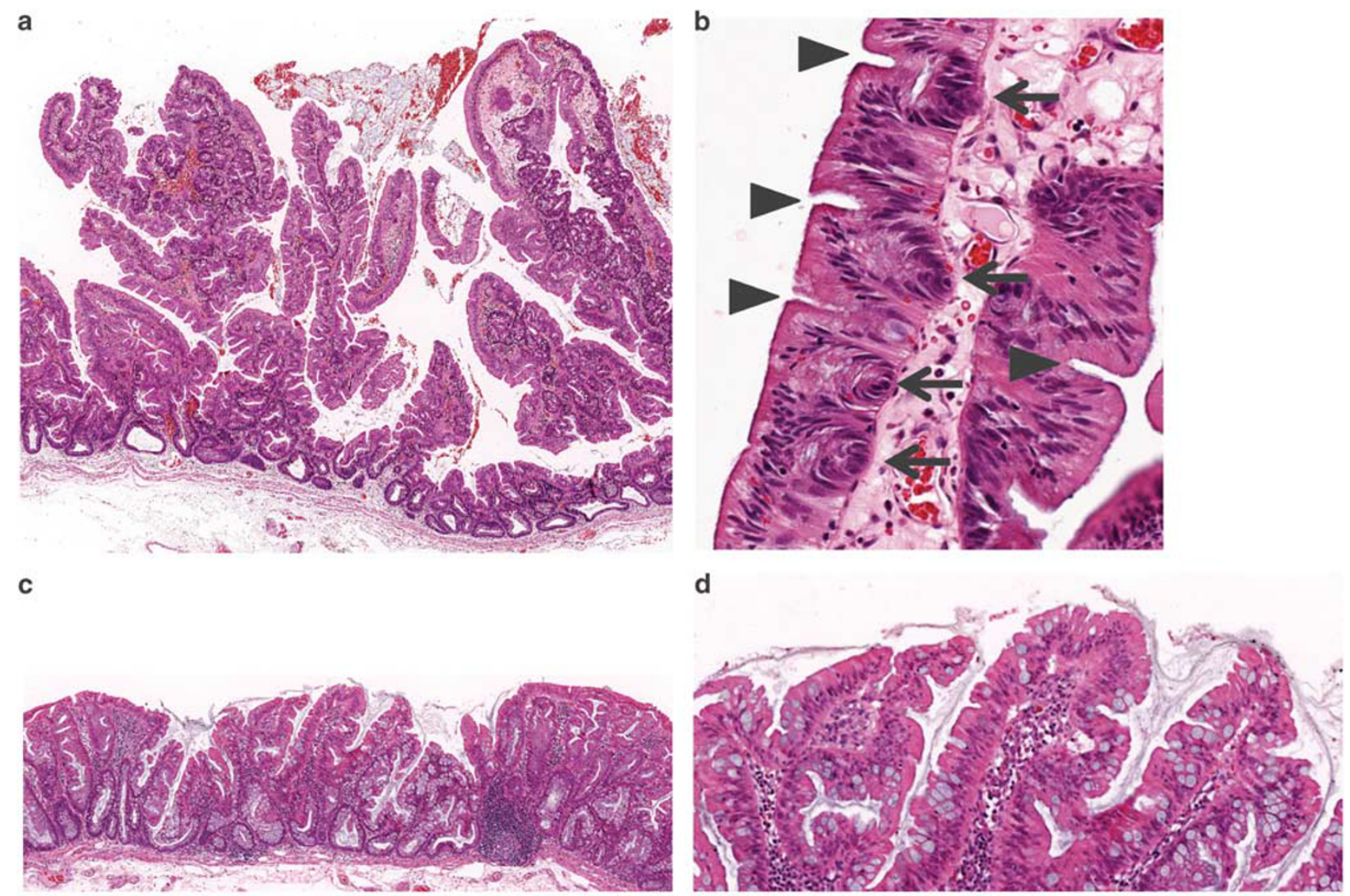

e

$\mathbf{f}$
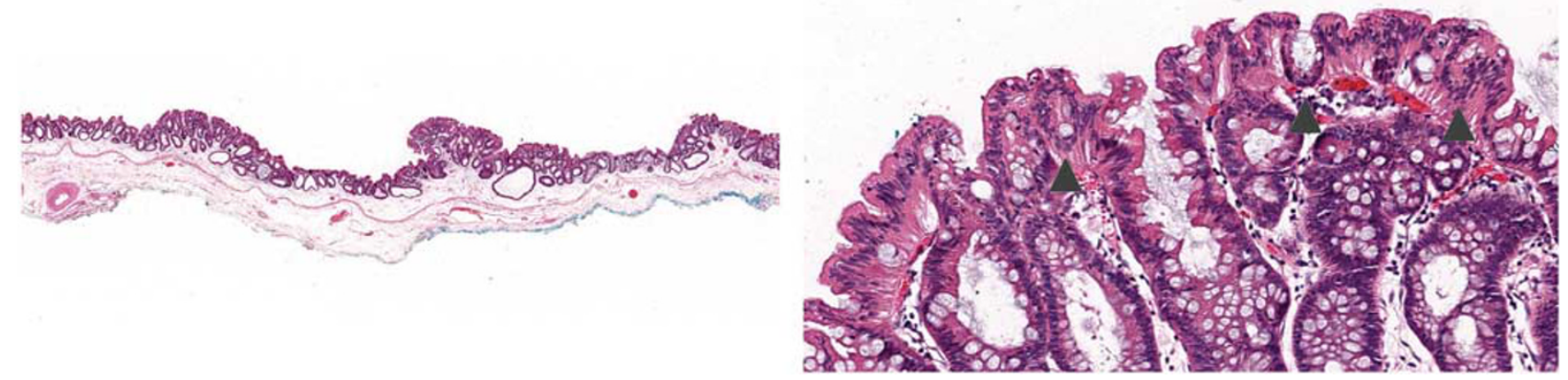

Figure 1 (a, b) BRAF mutant traditional serrated adenoma from the rectum with typical villiform projections. (b) Higher power better demonstrating typical cytology, slit-like serrations (arrowheads), and ectopic crypt formations (arrows). (c, d) BRAF mutant traditional serrated adenoma from the sigmoid colon with flat growth pattern. Sessile serrated adenoma-type crypts underlie traditional serrated adenoma with typical cytology and slit-like serrations better demonstrated at higher power in (d). (e, f) KRAS mutant traditional serrated adenoma from the rectum with flat growth pattern. (f) Higher power of (e) showing ectopic crypt formations (arrowheads) and typical cytology in the surface epithelium.

epithelial serrations referred to narrow slits in the epithelium similar to normal small intestinal mucosa $^{3}$ (Figure 1b) and ectopic crypt formations referred to epithelial buds with their bases not seated adjacent to the muscularis mucosae (Figure 1b). ${ }^{13}$

\section{Diagnosis and Validation of Flat Growth Pattern}

Each polyp was assessed for growth pattern (flat versus protuberant). A flat growth pattern was diagnosed when the majority of the polyps was elevated less than twice the height of the normal mucosa and lacked prominent viliform projections (Figures 1c-f).

Because flat growth in a traditional serrated adenoma is controversial, we further validated the diagnostic reproducibility of this subtype. A panel of four gastrointestinal pathologists (MB, NW, CR, and IB), blinded to all clinicopathological information, independently assessed the flat traditional serrated adenomas along with 50 sessile serrated 
adenomas randomly selected from a previous study set. ${ }^{10}$ The diagnosis of traditional serrated adenoma was based on the criteria outlined above and the diagnosis of sessile serrated adenoma was based on previously published criteria. ${ }^{10,21}$

\section{Identification of a Precursor Component}

After reaching the inclusion criteria for the study, each traditional serrated adenoma initially identified as having a precursor component by the principal author (MB) was additionally assessed for the presence of a precursor polyp of any type by three additional pathologists (NW, CR, and IB). The precursor component was required to represent a discrete area of the lesion with clear morphological distinction from the traditional serrated adenoma component either at the edge or underlying the traditional serrated adenoma (Figures 2a-c). ${ }^{16}$ Diagnosis of a sessile serrated adenoma precursor required the presence of at least one unequivocal sessile serrated adenoma-type crypt. $^{10,21}$ A precursor component was diagnosed if there was consensus among all four pathologists.

\section{Diagnosis of Advanced Traditional Serrated Adenoma}

The cohort was divided into 'ordinary' and 'advanced' traditional serrated adenomas based on the presence or absence of dysplasia or carcinoma. Dysplasia required an abrupt transition from typical traditional serrated adenoma to overt cytological dysplasia (Figure 2d). Cytological features included increased nuclear size, frequent and atypical mitoses, nuclear crowding, complete loss of polarity, and pseudo-stratification with nuclei extending into the upper half of the neoplastic cell. ${ }^{11,14,15}$ Architectural features were crowding of glands, cribriform glands, and intraluminal necrosis. Carcinoma was recognized by breach of the muscularis mucosae by cytologically dysplastic cells in concert with desmoplastic stroma.

All advanced areas were assessed for serrated morphology. In areas of dysplasia we used the criteria outlined in the WHO of cuboidal cells with eosinophilic cytoplasm, vesicular nuclei and prominent nucleoli. ${ }^{11}$ For areas of carcinoma we used the criteria of Makinen ${ }^{22}$ and required five features for a diagnosis of serrated morphology.

\section{Immunohistochemistry}

Immunohistochemistry was performed from the formalin-fixed, paraffin-embedded blocks. Sections were cut at $4 \mu \mathrm{m}$ and then dewaxed and rehydrated. Antigen retrieval for MLH1, CK7, p16, and Ki67 was performed by incubation in high $\mathrm{pH}$ antigen retrieval solution (pH9.0, Dako, Glostrup, Denmark) at $112{ }^{\circ} \mathrm{C}$ for $7 \mathrm{~min}$. Antigen retrieval for $\beta$-catenin, p53, and CK20 was performed by incubation in low $\mathrm{pH}$ antigen retrieval solution (pH6.0, Biocare Medical, Concord, CA, USA) at $112{ }^{\circ} \mathrm{C}$ for $7 \mathrm{~min}$.

Sections were manually stained following the manufacturer's instructions. Antibodies used were: MLH1 (clone G168-15, 1:100, BD Pharmingen, Franklin Lakes, NJ, USA), $\beta$-catenin (1:600, Cell Marque, Rocklin, CA, USA), p53 (clone DO-7, 1:150, Biocare Medical), p16 (clone JC8, 1:150, Santa Cruz Biotechnology, Dallas, TX, USA), Ki67 (clone MIB-1, 1:100, Dako), CK7 (clone OV-TL12/30, 1:100, Dako), and CK20 (clone Ks20.8, 1:150, Biocare Medical). Slides were counterstained with Mayer's hematoxylin.

Each marker was assessed for both intensity and extent of staining in the following compartments: basal zone, typical eosinophilic cells, ectopic crypt formations, goblet cells, dysplastic components, and invasive carcinoma components. Intensity was scored as $0-3(0=$ no staining, $1=$ weak staining, $2=$ moderate staining, $3=$ strong staining) and extent as $0-4 \quad(0=$ no staining, $1=1-10 \%$ of cells, $2=11-50 \%$ of cells, $3=51-90 \%$ of cells, $4=>90 \%$ of cells). When intensity was variable, an average of the intensity was used. A final score was determined by multiplying the intensity and extent scores (minimum score $=0$, maximum $=12$ ).

Interpretation of each marker was as follows. Normal MLH1 expression required the presence of nuclear staining with a score of $\geq 3$ in each compartment. Positive $\beta$-catenin required nuclear staining with a score of $\geq 2$ in any compartment. Loss of membrane staining and cytoplasmic staining were not considered positive. ${ }^{14,23}$ Positive p53 required nuclear staining with a score of $\geq 6$ in any compartment. ${ }^{14}$ Positive P16 required either cytoplasmic or nuclear staining with a score of $\geq 3$ in any compartment. ${ }^{24}$ Positive Ki67 required nuclear staining with a score of $\geq 9$ in any compartment. Positive cytokeratin 7 and cytokeratin 20 required cytoplasmic staining with a score of $\geq 3$ in any compartment. For ordinary traditional serrated adenomas, the compartment with the highest score was used for analysis. For advanced traditional serrated adenomas, the score only in the areas of dysplasia or carcinoma was used for analysis.

\section{DNA Extraction}

DNA was extracted from the formalin-fixed, paraffin-embedded blocks using the Chelex-100 extraction method (Bio-Rad Laboratories, Hercules, CA, USA). In brief, three $10 \mu \mathrm{m}$ sections were cut from the FFPE blocks and heated to $90{ }^{\circ} \mathrm{C}$ in $200 \mu \mathrm{l}$ of $0.5 \%$ Tween-20 in $1 \times \mathrm{TE}$ and then digested with $80 \mathrm{mg}$ of proteinase $\mathrm{K}$ at $55^{\circ} \mathrm{C}$ for $3 \mathrm{~h}$. After digestion, $200 \mu \mathrm{l}$ of $5 \%$ Chelex-100 was added to the samples and they were heated to $99^{\circ} \mathrm{C}$, centrifuged then cooled on ice, and the paraffin layer removed. Then, $200 \mu \mathrm{l}$ of chloroform was added, the 


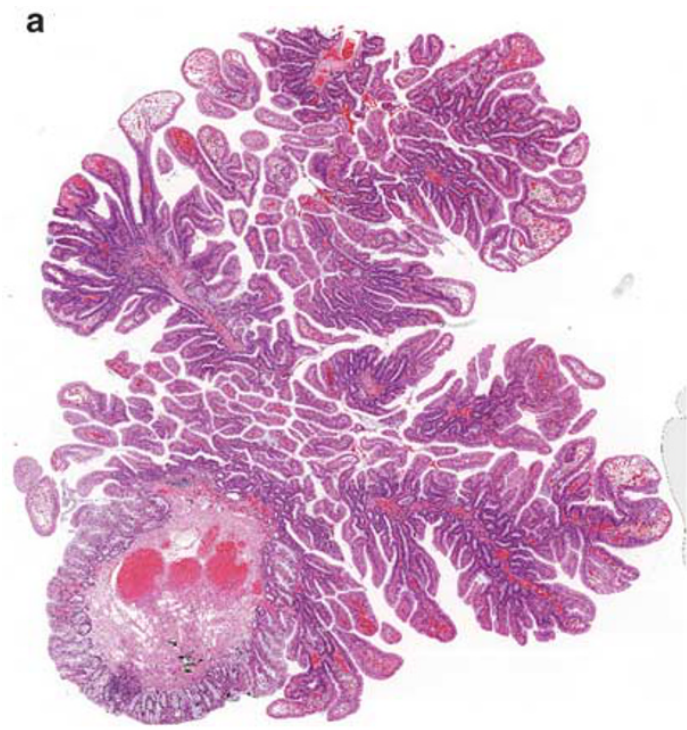

b
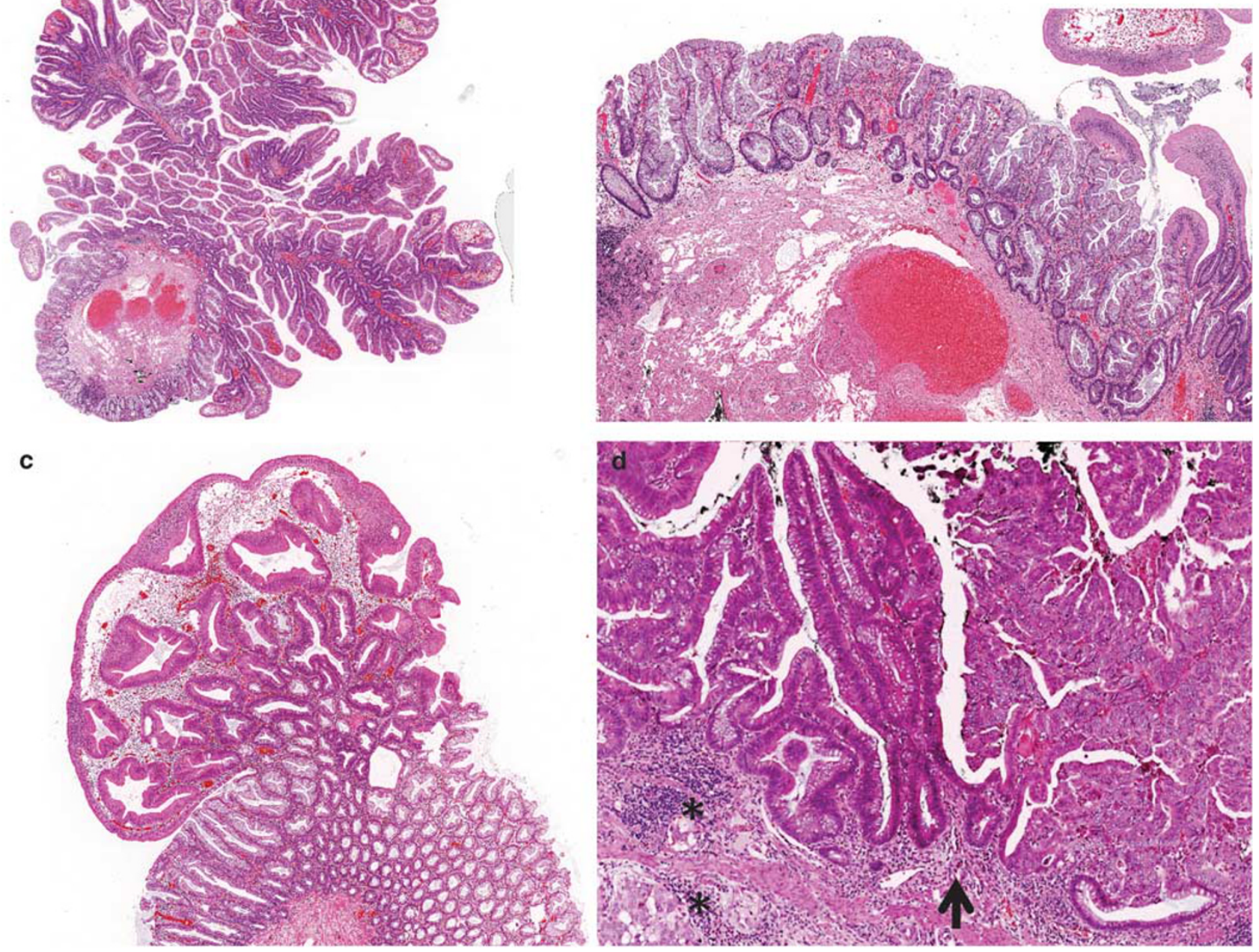

Figure $2(\mathbf{a}, \mathbf{b})$. A protuberant $B R A F$ mutant traditional serrated adenoma from the sigmoid colon with adjacent sessile serrated adenoma better demonstrated at higher power in (b). (c) A small but protuberant BRAF mutant traditional serrated adenoma from the rectum arising from a microvesicular hyperplastic polyp. (d) An advanced BRAF mutant traditional serrated adenoma from the transverse colon (left) with abrupt transition (arrow) to high-grade serrated dysplasia (right). This polyp also had a small focus of invasive carcinoma (not shown); however, note the carcinoma within the lymphatics of the mucosa and submucosa (asterisks).

samples centrifuged for $15 \mathrm{~min}$, and the final product from the surface phase removed by manual pipette. DNA concentration was established by spectrophotometry (NanoDrop 2000, Thermo Scientific, Fremont, CA, USA). In cases where there was contamination of the formalin-fixed, paraffinembedded block by nonpolypoid tissue, manual microdissection was performed using a sterile scalpel blade with a marked hematoxylin and eosin-stained section as a guide.

\section{BRAF and KRAS Mutation Detection}

The BRAF V600E mutation was detected by allelespecific PCR as previously described. ${ }^{25}$ KRAS mutations were assessed by high-resolution melt analysis as previously described. ${ }^{26}$

\section{CpG Island Methylator Phenotype Determination}

Polyp genomic DNA was treated with sodium bisulfite using the Epitect Fast Bisulfite Conversion Kit (Qiagen, Duesseldorf, Germany) according to the manufacturer's instructions. CpG island methylator phenotype status was determined using the MethyLight technique as previously described by Weisenberger et $a l^{27}$ The CpG island methylator phenotype panel genes consisted of CACNA1G, IGF2, NEUROG1, RUNX3, and SOCS1. The output from this assay was percent of sample DNA methylated relative to a DNA reference sample, to give a percentage of methylated reference. Using previously published criteria, ${ }^{27}$ samples with $\geq 3$ markers with a percentage of methylated reference of $>10$ were considered $\mathrm{CpG}$ island methylator 
phenotype high, samples with one or two markers with a percentage of methylated reference $>10$ were considered $\mathrm{CpG}$ island methylator phenotype low, and samples with no markers $>10$ were considered $\mathrm{CpG}$ island methylator phenotype negative. Completely methylated genomic DNA from pooled blood samples was used to generate a standard curve for each gene. Negative controls were run on each plate and the ALU gene was used to ensure the quality of each sample of bisulfite-treated DNA. As previously published, we considered samples to have failed if the ALU cycle threshold value (Ct) was $>23 .^{28}$ In addition, we required the ALU representative calculated concentration to be $>1000$. The methylation status of the MLH1 promoter region was also assessed using MethyLight using the same technique as described above. The probe and primer sequences are as described previously. ${ }^{29}$

\section{Statistical Analysis}

Categorical variables were compared by Fisher's exact test and continuous variables by Student's $t$-test. A $P$-value of $\leq 0.05$ was considered significant. Interrater agreements of diagnoses by different pathologists were estimated using Fleiss's $\kappa$ and intraclass correlation coefficients were also obtained. SPSS version 19, R version 3.0.2, and GraphPad Prism version 6.02 were used for statistical analyses.

\section{Results}

\section{Clinicopathological Data}

The clinicopathological data are presented according to the presence or absence of advanced histology and by mutation status in Tables 1 and 2. Advanced traditional serrated adenomas were larger (mean $25 \mathrm{~mm}$ ) and less often associated with a precursor polyp (13\%) than ordinary traditional serrated adenomas (mean $16 \mathrm{~mm}, 38 \%$ precursor polyp). Most traditional serrated adenomas had mutation of either BRAF (67\%) or KRAS $(22 \%)$ and these mutations were mutually exclusive (Table 2). BRAF mutant traditional serrated adenomas were more often proximal (39\%) and had more frequent origin in a precursor polyp (57\%) than KRAS mutant traditional serrated adenomas that were rarely proximal $(2 \%)$ and never associated with a precursor polyp (Table 2).

Table 3 outlines the inclusion criteria relative to polyp size. Smaller polyps were less likely to have ectopic crypt formations. Similarly, 19 of 76 (25\%) flat traditional serrated adenomas did not have ectopic crypt formations; in contrast, only 3 of 124 $(2 \%)$ protuberant traditional serrated adenomas did not show ectopic crypt formations $(P<0.0001)$. Table 4 demonstrates the relationship between polyp location, mutation status, and flat morphol- ogy. BRAF mutant and proximal polyps were more likely to be flat than distal polyps. However, after accounting for anatomical location, no difference was observed by mutation status alone. Reproducibility of the diagnosis of flat traditional serrated adenomas compared with sessile serrated adenomas was excellent, with a $\kappa$-value of $0.93(P<0.001)$ and the intraclass correlation coefficients absolute agreement of 0.981 (95\% confidence interval 0.9750.986; $P<0.001)$.

All advanced areas were assessed for serrated morphology (Table 5). The majority of cases had serrated morphology. All cases with both dysplasia and invasive carcinoma showed the same morphology in both components. No significant associations could be identified between serrated morphology and molecular or immunohistochemical profile.

\section{Mutation Status and the CpG Island Methylator Phenotype}

$B R A F$ and $K R A S$ mutation were present in $67 \%$ and $22 \%$ of polyps, respectively. $B R A F$ mutant, KRAS mutant, and $B R A F / K R A S$ wild-type polyps were $\mathrm{CpG}$ island methylator phenotype high in $60 \%$, $16 \%$, and $17 \%$ respectively, low in $28 \%, 44 \%$, and $48 \%$, respectively, and negative in $11 \%, 40 \%$, and $35 \%$, respectively. The control tubulovillous adenomas were $\mathrm{CpG}$ island methylator phenotype high, low, and negative in $0 \%, 6 \%$, and $94 \%$, respectively. $B R A F$ mutation was more frequent than KRAS mutation $(P<0.0001)$ and was more often associated with $\mathrm{CpG}$ island methylator phenotype high than KRAS mutation $(P<0.0001)$; however, KRAS mutant traditional serrated adenomas were more frequently CpG island methylator phenotype high than control tubulovillous adenomas $(P=0.0034)$. In all, $75 \%$ (44/59) of proximal traditional serrated adenomas were $\mathrm{CpG}$ island methylator phenotype high, compared with $35 \%(48 / 139)$ of distal traditional serrated adenomas $(P<0.0001)$ and, in addition, $83 \%(43 / 59)$ of proximal $B R A F$ mutant traditional serrated adenomas were $\mathrm{CpG}$ island methylator phenotype high, compared with 46\% (38/82) of distal $B R A F$ mutant traditional serrated adenomas $(P<0.0001)$. Despite this, distal $B R A F$ mutant traditional serrated adenomas remained more likely to be CpG island methylator phenotype high than distal KRAS mutant traditional serrated adenomas $(P=0.0025)$. The $\mathrm{CpG}$ island methylator phenotype-low status was present in $44 \%$ and $48 \%$ of $K R A S$ mutant and $B R A F / K R A S$ wild-type traditional serrated adenomas, respectively, but was seen in only $6 \%$ of control tubulovillous adenomas. Advanced traditional serrated adenomas showed no statistically significant association with mutation status or $\mathrm{CpG}$ island methylator phenotype when compared with ordinary traditional serrated adenomas. No BRAF mutations were identified in the control tubulovillous adenomas. 
Table 1 Clinicopathological features by advanced histology

\begin{tabular}{|c|c|c|c|c|}
\hline & $\begin{array}{c}\text { All traditional } \\
\text { serrated } \\
\text { adenomas }(\mathrm{n}=200)\end{array}$ & $\begin{array}{l}\text { Ordinary traditional } \\
\text { serrated } \\
\text { adenomas }(\mathrm{n}=162)\end{array}$ & $\begin{array}{c}\text { Advanced traditional } \\
\text { serrated } \\
\text { adenomas }(\mathrm{n}=38)\end{array}$ & $\begin{array}{c}\text { P-value } \\
\text { (ordinary } \\
\text { versus advanced) }\end{array}$ \\
\hline Age & $64(27-89)$ & $64(27-89)$ & $65(27-85)$ & 0.8069 \\
\hline Female & $50 \%$ & $51 \%$ & $45 \%$ & 0.5891 \\
\hline Mean size (mm) & 16 (3-95) (median 12) & 14 (3-95) (median 11) & $25(5-70)($ median 21$)$ & $<0.0001$ \\
\hline Distal location & $71 \%$ & $68 \%$ & $82 \%$ & 0.1153 \\
\hline Precursor polyp & $38 \%$ & $44 \%$ & $13 \%$ & 0.0003 \\
\hline Sessile serrated adenoma & $31 \%$ & $36 \%$ & $11 \%$ & 0.0018 \\
\hline Microvesicular hyperplastic polyp & $7 \%$ & $8 \%$ & $3 \%$ & 0.4769 \\
\hline
\end{tabular}

$P$-values $<0.05$ are indicated in bold.

Table 2 Clinicopathological features by mutation status

\begin{tabular}{|c|c|c|c|c|}
\hline Feature & $\begin{array}{l}\text { BRAF mutation } \\
\quad(\mathrm{n}=134)\end{array}$ & $\begin{array}{l}\text { KRAS mutation } \\
\quad(\mathrm{n}=43)\end{array}$ & $\begin{array}{c}B R A F / K R A S \\
\text { wild type }(\mathrm{n}=23)\end{array}$ & $\begin{array}{c}\mathrm{P} \text {-value (BRAF } \\
\text { versus KRAS) }\end{array}$ \\
\hline Age & $64(27-89)$ & $65(36-86)$ & $62(36-87)$ & 0.8611 \\
\hline Female & $49 \%$ & $49 \%$ & $57 \%$ & 1.000 \\
\hline Mean size (mm) & $14(3-70)($ median 12$)$ & $18(3-60)$ (median 13) & 20 (4-95) (median 13) & 0.0550 \\
\hline Distal location & $61 \%$ & $98 \%$ & $74 \%$ & $<0.0001$ \\
\hline Precursor polyp & $57 \%$ & $0 \%$ & $0 \%$ & $<0.0001$ \\
\hline Sessile serrated adenoma & $46 \%$ & $0 \%$ & $0 \%$ & $<0.0001$ \\
\hline Microvesicular hyperplastic polyp & $10 \%$ & $0 \%$ & $0 \%$ & 0.0233 \\
\hline
\end{tabular}

$P$-values $<0.05$ are indicated in bold.

Table 3 Inclusion criteria by polyp size

\begin{tabular}{|c|c|c|c|c|c|c|c|c|c|}
\hline \multirow{2}{*}{ Size } & \multicolumn{3}{|c|}{ Ectopic crypt formations } & \multicolumn{3}{|c|}{ Slit-like serrations } & \multicolumn{3}{|c|}{ Typical cytology } \\
\hline & Absent & $<50 \%$ & $>50 \%$ & Absent & $<50 \%$ & $>50 \%$ & Absent & $<50 \%$ & $>50 \%$ \\
\hline$<10 \mathrm{~mm}(n=65)$ & $22 \%$ & $49 \%$ & $29 \%$ & $2 \%$ & $45 \%$ & $54 \%$ & 0 & $5 \%$ & $95 \%$ \\
\hline$>10 \mathrm{~mm}(n=135)$ & $6 \%$ & $41 \%$ & $53 \%$ & $2 \%$ & $44 \%$ & $54 \%$ & 0 & $4 \%$ & $96 \%$ \\
\hline$P$-value $(<10$ versus $\geq 10 \mathrm{~mm})$ & 0.0028 & 0.2880 & 0.0015 & 1.0000 & 1.0000 & 1.0000 & 1.0000 & 1.0000 & 1.0000 \\
\hline
\end{tabular}

$P$-values $<0.05$ are indicated in bold.

Table 4 Flat morphology relative to location and mutation status

\begin{tabular}{lccc}
\hline Flat morphology & All cases & Proximal location & Distal location \\
\hline All cases & $76 / 200(38 \%)$ & $37 / 59(63 \%)$ & $39 / 141(28 \%)$ \\
BRAF mutant & $57 / 134(43 \%)$ & $33 / 52(64 \%)$ & $24 / 82(29 \%)$ \\
KRAS mutant & $10 / 41(24 \%)$ & $0 / 1(0 \%)$ & $10 / 4025 \%$ \\
Wild type & $9 / 23(39 \%)$ & $4 / 6(67 \%)$ & $5 / 17(29 \%)$ \\
$P$-value (BRAF versus KRAS mutant) & $\mathbf{0 . 0 4 3 6}$ & 0.3774 & 0.6726 \\
\hline
\end{tabular}

$P$-values $<0.05$ are indicated in bold.

${ }^{\mathrm{a}}$ Location was not available for two of the KRAS mutant TSAs.

MLH1 promoter methylation was present in $7 \%$ (9/134) of the BRAF mutant traditional serrated adenomas (8 ordinary and 1 advanced), but in none of the KRAS mutant or BRAF/KRAS wild-type traditional serrated adenomas. Only the single advanced traditional serrated adenoma with MLH1 methylation showed concordant loss of MLH1 expression by immunohistochemistry.

\section{Immunohistochemistry}

Table 6 outlines the immunohistochemical features of the study polyps. Among the ordinary traditional serrated adenomas, BRAF mutant traditional serrated adenomas were more likely than KRAS mutant traditional serrated adenomas to have a high Ki67 proliferative index in the basal compartment. No 
Table 5 Morphology of the advanced traditional serrated adenomas

\begin{tabular}{lcc}
\hline & $\begin{array}{c}\text { Conventional } \\
\text { morphology }\end{array}$ & $\begin{array}{c}\text { Serrated } \\
\text { morphology }\end{array}$ \\
\hline BRAF mutant & & \\
Dysplastic component & $4 / 19$ & $15 / 19$ \\
Invasive component & $2 / 9$ & $7 / 9$ \\
KRAS mutant & $3 / 7$ & $4 / 7$ \\
Dysplastic component & $0 / 4$ & $4 / 4$ \\
Invasive component & & \\
BRAF/KRAS wild type & $1 / 6$ & $5 / 6$ \\
Dysplastic component & $0 / 2$ & $2 / 2$ \\
Invasive component & & \\
\hline
\end{tabular}

The study cohort included 38 advanced traditional serrated adenomas, including 22 with $B R A F$ mutation, 10 with KRAS mutation, and $6 B R A F / K R A S$ wild type. In some polyps, both dysplasia and carcinoma were present and these components were assessed separately. In all cases displaying both dysplasia and carcinoma, the two components had the same morphology.

other statistically significant differences in staining patterns were identified between the ordinary traditional serrated adenomas when stratified according to mutation status. Advanced traditional serrated adenomas showed significantly increased nuclear staining for $\beta$-catenin (Figures $3 \mathrm{a}$ and $\mathrm{b}$ ) and p53 (Figure 3c) compared with the ordinary traditional serrated adenomas. MLH1 nuclear staining was retained in all but one case. P16 staining was lost in $42 \%$ of the dysplastic components and $89 \%$ of the invasive components in advanced $B R A F$ mutant polyps (Figure 3d). In contrast, p16 staining was lost in only $8 \%$ of dysplastic and $17 \%$ of invasive components in the KRAS mutant and $B R A F / K R A S$ wild-type polyps.

\section{Discussion}

This clinicopathological, morphological, and molecular appraisal of a large series of rigorously categorized traditional serrated adenomas was undertaken with the aim of clarifying areas of morphological and molecular uncertainty related to these polyps. We focused on identifying features that discriminated BRAF and KRAS mutant cases and on identifying pathways by which traditional serrated adenomas progress to carcinoma.

Because of the variability in traditional serrated adenoma morphology and its evolution as they enlarge, absolute diagnostic criteria are difficult to define, although typical cytology, ectopic crypt formations, slit-like luminal serrations, and protuberant or villiform growth have proven useful. Slit-like serrations are possibly the most specific, differentiating traditional serrated adenomas from morphologically similar tubulovillous adenomas with prominent serration. ${ }^{30}$ To ensure all polyps in the study group were traditional serrated adenomas, we required all cases to show at least two of the first three features listed, with at least one present in $50 \%$ of the polyp. Table 3 shows the relative contribution of each of these features. Of note, smaller polyps are less likely to show ectopic crypt formations.

We specifically did not include protuberant or viliform growth as an inclusion criterion, as we regularly identify traditional serrated adenomas with a flat growth pattern. Because the concept of a flat traditional serrated adenoma is contentious, we performed a reproducibility study to determine whether we could reliably distinguish these polyps from sessile serrated adenomas. In this study we achieved an excellent level of interobserver concordance, indicating that flat traditional serrated adenomas can be reliably distinguished from sessile serrated adenomas. Flat traditional serrated adenomas are typically (but not exclusively) proximal, $B R A F$ mutant and arise in sessile serrated adenomas. Although proximal polyps are more likely to be flat, this is unrelated to mutation status, with distal $B R A F$ and KRAS mutant traditional serrated adenomas showing similar rates of flat and protuberant growth. This correlates with a previous study showing that distal sessile serrated adenomas can also be protuberant, ${ }^{31}$ thus flat versus protuberant morphology is probably secondary to location rather than being an intrinsic element of the traditional serrated adenoma.

Our clinicopathological data are in agreement with recent publications, ${ }^{14-17}$ although in our series, precursor polyps were limited to microvesicular hyperplastic polyps and sessile serrated adenomas and were exclusively associated with $B R A F$ mutation. BRAF mutant traditional serrated adenomas were also more likely to be proximal than KRAS or BRAF/KRAS wild-type traditional serrated adenomas. Other than the association with a precursor polyp, no differences in morphology were identified based on mutation status alone.

A critical morphological issue is the distinction between traditional serrated adenoma arising in a sessile serrated adenoma from a sessile serrated adenoma with cytological dysplasia. Based on our data, there are important biological differences between these two lesions, particularly relating to mismatch repair enzyme function, and thus histological distinction is important. When the traditional serrated adenoma component is obvious, the diagnosis should not be difficult; however, it is fairly common to see a small but discrete focus with traditional serrated adenoma-type cytology arising in an otherwise typical sessile serrated adenoma. In our opinion, this may represent senescent change, or possibly early traditional serrated adenoma arising in the sessile serrated adenoma. Some pathologists consider such change as serrated dysplasia, warranting a diagnosis of sessile serrated adenoma with dysplasia. In an audit of our own practice, a subset 
Table 6 Immunohistochemistry results of ordinary and advanced traditional serrated adenomas and control tubulovillous adenomas

\begin{tabular}{|c|c|c|c|c|}
\hline Antibody & $\begin{array}{c}\text { Ordinary traditional serrated } \\
\text { adenoma }(\mathrm{n}=162)\end{array}$ & $\begin{array}{c}\text { Advanced traditional serrated } \\
\text { adenoma }(\mathrm{n}=38)\end{array}$ & $\begin{array}{l}\text { Tubulovillous } \\
\text { adenoma }(\mathrm{n}=50)\end{array}$ & $\begin{array}{l}\text { P-value (ordinary } \\
\text { versus advanced) }\end{array}$ \\
\hline \multicolumn{5}{|l|}{ MLH1 $\operatorname{loss}^{\mathrm{a}}$} \\
\hline All polyps & $0 \%$ & $3 \%$ & $0 \%$ & 0.35 \\
\hline$B R A F$ mutant & $0 \%$ & $5 \%$ & & 0.31 \\
\hline KRAS mutant & $0 \%$ & $0 \%$ & & 1.000 \\
\hline$B R A F / K R A S$ wild type & $0 \%$ & $0 \%$ & & 1.000 \\
\hline \multicolumn{5}{|l|}{ Nuclear $\beta$-catenin ${ }^{\mathrm{a}}$} \\
\hline All polyps & $7 \%$ & $39 \%$ & $84 \%$ & $<0.0001$ \\
\hline$B R A F$ mutant & $5 \%$ & $32 \%$ & & 0.0011 \\
\hline KRAS mutant & $12 \%$ & $60 \%$ & & 0.0048 \\
\hline$B R A F / K R A S$ wild type & $6 \%$ & $50 \%$ & & 0.0401 \\
\hline \multicolumn{5}{|l|}{$P 53^{\mathrm{a}}$} \\
\hline All polyps & $6 \%$ & $55 \%$ & $10 \%$ & $<0.0001$ \\
\hline$B R A F$ mutant & $7 \%$ & $45 \%$ & & $<0.0001$ \\
\hline KRAS mutant & $0 \%$ & $70 \%$ & & $<0.0001$ \\
\hline$B R A F / K R A S$ wild type & $6 \%$ & $67 \%$ & & 0.0078 \\
\hline \multicolumn{5}{|l|}{$P 16^{\mathrm{a}}$} \\
\hline All polyps & $73 \%$ & $63 \%$ & $86 \%$ & 0.24 \\
\hline$B R A F$ mutant & $74 \%$ & $45 \%$ & & 0.0113 \\
\hline KRAS mutant & $76 \%$ & $90 \%$ & & 0.6591 \\
\hline$B R A F / K R A S$ wild type & $59 \%$ & $83 \%$ & & 0.3690 \\
\hline \multicolumn{5}{|l|}{$K i 67^{\mathrm{a}}$} \\
\hline All polyps & $53 \%$ & $84 \%$ & $92 \%$ & 0.0004 \\
\hline$B R A F$ mutant & $59 \%$ & $82 \%$ & & 0.0056 \\
\hline KRAS mutant & $33 \%$ & $90 \%$ & & 0.0027 \\
\hline$B R A F / K R A S$ wild type & $53 \%$ & $83 \%$ & & 0.3401 \\
\hline \multicolumn{5}{|l|}{$C K 7^{\mathrm{a}}$} \\
\hline All polyps & $38 \%$ & $32 \%$ & $16 \%$ & 0.5758 \\
\hline$B R A F$ mutant & $40 \%$ & $36 \%$ & & 0.8147 \\
\hline KRAS mutant & $48 \%$ & $20 \%$ & & 0.1529 \\
\hline$B R A F / K R A S$ wild type & $0 \%$ & $33 \%$ & & 0.0593 \\
\hline \multicolumn{5}{|l|}{$C K 20^{\mathrm{a}}$} \\
\hline All polyps & $98 \%$ & $79 \%$ & $92 \%$ & 0.0002 \\
\hline$B R A F$ mutant & $97 \%$ & $73 \%$ & & 0.0006 \\
\hline KRAS mutant & $97 \%$ & $90 \%$ & & 0.2874 \\
\hline$B R A F / K R A S$ wild type & $100 \%$ & $83 \%$ & & 0.2609 \\
\hline
\end{tabular}

${ }^{\mathrm{a}}$ Immunohistochemical scoring as per Materials and methods.

of sessile serrated adenomas with dysplasia (mostly before 2012) showed this change (Mark Bettington and Neal Walker, unpublished data). In our more recent opinion, rendering a diagnosis of sessile serrated adenoma with dysplasia in this situation will imply a significantly greater degree of malignant risk than is warranted. True sessile serrated adenomas with dysplasia have been demonstrated to show Wnt pathway activation, ${ }^{23}$ loss of mismatch repair function, ${ }^{32}$ CDKN2A silencing, ${ }^{24}$ and sometimes TP53 mutation. ${ }^{33}$ In contrast, from our data, ordinary traditional serrated adenomas arising in a sessile serrated adenoma do not have these advanced features. Thus, in our opinion, calling these lesions sessile serrated adenoma with dysplasia risks diminishing the significance of true sessile serrated adenoma with dysplasia and potentially leads to erroneous conclusions regarding surveillance.
The final important morphological point is the diagnosis of dysplasia arising in a traditional serrated adenoma. In our series we found a very high rate of both serrated type dysplasia and carcinoma among our advanced polyps. This is in keeping with the work of Makinen et $a,^{22,34}$ who defined serrated adenocarcinoma primarily from cancers arising in traditional serrated adenomas. In a subsequent paper, Stefanius et $a l^{35}$ showed that both KRAS and BRAF mutations are common in serrated adenocarcinomas. Our findings are in keeping with these earlier studies but are different to those more recently published by Tsai et al. ${ }^{15}$

In addition, we are of the opinion that the typical traditional serrated adenoma cytology does not represent a serrated dysplastic change. ${ }^{11}$ This is a controversial issue; the reasons for our opinion are as follows. First, the typical traditional serrated 

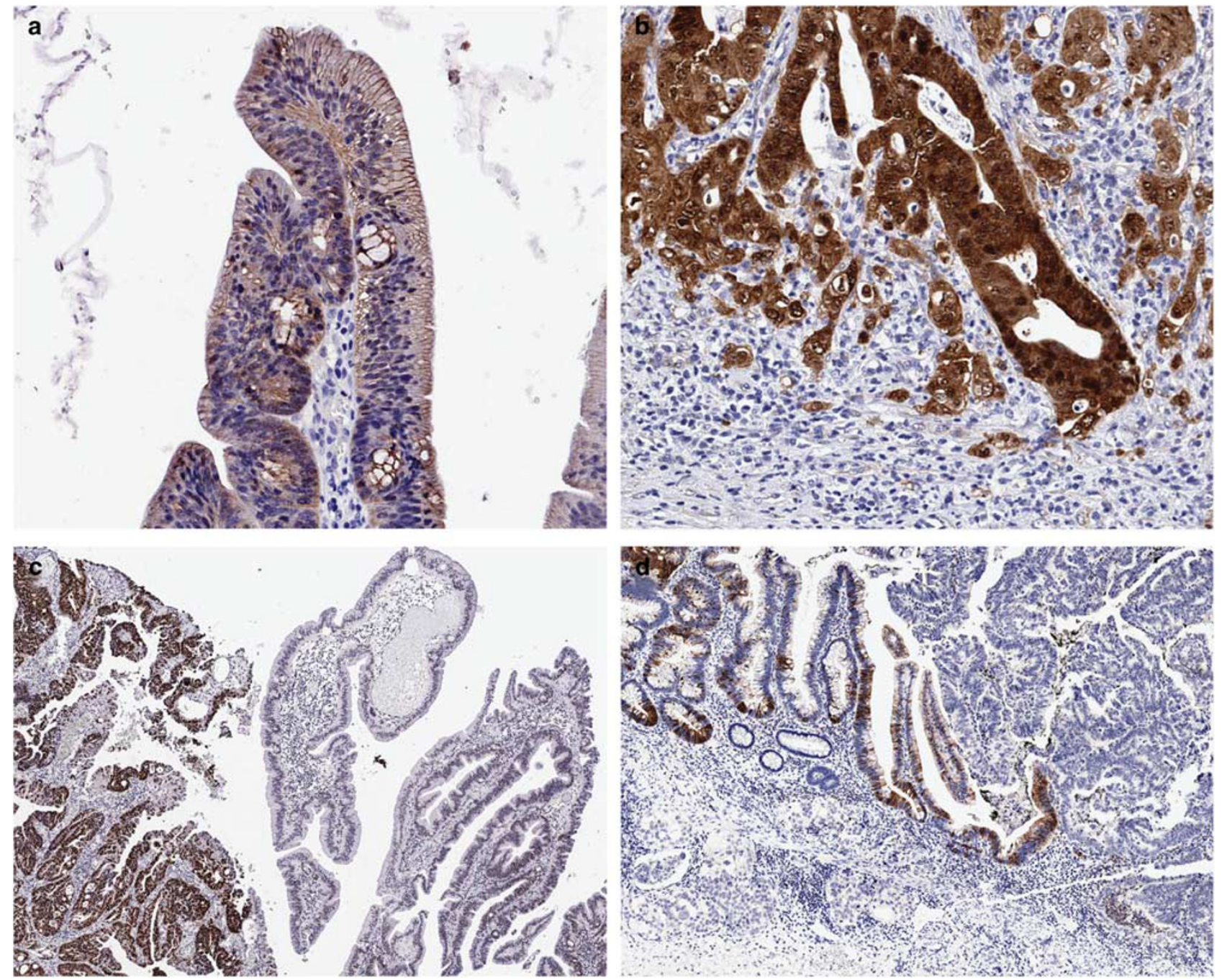

Figure 3 (a) High power of the normal $\beta$-catenin staining pattern in an ordinary traditional serrated adenoma. Note the distinct membrane staining without cytoplasmic or nuclear staining, including in the ectopic crypt formations. (b) Abnormal pattern of $\beta$-catenin in an area of carcinoma showing strong nuclear and cytoplasmic staining. (c) Traditional serrated adenoma showing strong nuclear p53 staining in the advanced component (left) and lack of staining in the ordinary component (right). (d) Traditional serrated adenoma showing positive staining for p16 in the ordinary component (left), particularly in the proliferative basal compartment and abrupt loss of staining in the advanced component (right).

adenoma cytology is not overtly atypical; second, these cells do not show mitoses and have a very low proliferative index by Ki67 staining; and third, they do not show the advanced features seen in the overtly dysplastic cells of advanced polyps, namely positive nuclear staining for $\beta$-catenin and p53 and loss of staining for p16. Instead of routinely describing dysplasia in traditional serrated adenomas, we propose that the nomenclature be brought into line with that of the sessile serrated adenoma, whereby the ordinary traditional serrated adenoma is simply designated traditional serrated adenoma, with no mention of cytological dysplasia, and advanced traditional serrated adenomas are designated traditional serrated adenoma with dysplasia or carcinoma as appropriate. Although different patterns of dysplasia occur, the key point is to identify traditional serrated adenomas with advanced biology and a higher risk of malignant progression as evidenced by the Wnt pathway activation, TP53 mutation, and $C D K N 2 A$ loss demonstrated in this study. Thus, similar to the sessile serrated adenoma, reporting the presence of overt dysplasia is the critical issue. At this time, assigning a grade or dividing dysplasia into serrated versus conventional types has no clinical utility and may only introduce confusion into the nomenclature.

The second major aim of this paper was to give a thorough account of the molecular features of the traditional serrated adenoma. To this end, we performed BRAF, KRAS, and CpG island methylator phenotype analysis on all polyps in the series. In line with recent publications, ${ }^{16,17}$ we found that $B R A F$ mutant traditional serrated adenomas represented about two-thirds of all cases and the vast majority of proximal traditional serrated adenomas. 
In contrast, KRAS mutant traditional serrated adenomas were almost exclusively distal, with a particular predilection for the rectum. BRAF/KRAS wild-type cases were more closely aligned with KRAS mutant polyps than BRAF mutant cases and may have as yet undefined upregulation of MAP kinase signaling.

CpG island methylator phenotype-high status, as expected, correlated strongly with both proximal location and BRAF mutation. However, $16 \%$ of KRAS mutant traditional serrated adenomas were CpG island methylator phenotype high and 44\% were $\mathrm{CpG}$ island methylator phenotype low, indicating that methylation may still play an important role in the malignant progression of these polyps. In addition, the level of $\mathrm{CpG}$ island methylator phenotype in the KRAS mutant and BRAF/KRAS wild-type traditional serrated adenoma was significantly more than seen in the control tubulovillous adenomas.

Some authors have questioned the validity of including traditional serrated adenomas as part of the serrated neoplasia pathway; however, the combination of MAP kinase pathway activation and CpG island methylator phenotype is strong evidence to support their continued inclusion. Part of this confusion may stem from the lack of reliability of CpG island methylator phenotype data reported in the literature. Unfortunately, no uniform panel for the $\mathrm{CpG}$ island methylator phenotype assessment has been utilized. In addition, in many studies, no controls were included or the control cases show the CpG island methylator phenotype far outside of what could reasonably be expected based on the reported histological diagnosis. ${ }^{15,19,20,36}$ This calls into question the $\mathrm{CpG}$ island methylator phenotype results for some of these studies. In the present study, we used the well-validated panel described by Weisenberger et $a .^{27}$ In this series, the vast majority of control tubulovillous adenomas were CpG island methylator phenotype negative (94\%) and none were CpG island methylator phenotype high, and this is the expected outcome and provides strong support for the validity of our results.

The final aim of this study was to interrogate the pathways by which traditional serrated adenomas progress to carcinoma. We stained each polyp with seven immunohistochemical markers. The ordinary traditional serrated adenomas were remarkably uniform in their staining patterns. Ki67 and cytokeratin 20 predominantly showed the pattern described by Torlakovic et al, with a high Ki67 index in the ectopic crypt formations and basal crypts but very limited in the typical surface cells and the opposite pattern with cytokeratin $20 .{ }^{13}$ The Ki67 index was high in essentially all areas of dysplasia or early carcinoma. A proportion of ordinary and advanced traditional serrated adenomas, particularly $B R A F$ mutant cases, showed an aberrant cytokeratin 7 and cytokeratin 20 immunophenotype. A previous study of BRAF mutant colorectal carcinomas showed similar aberrations, ${ }^{37}$ but were most evident in the microsatellite-unstable group. This knowledge can be important when working up malignancies of unknown origin, but is not informative in the diagnosis of individual polyps.

Perhaps the most important feature of the advanced traditional serrated adenomas was the almost uniform retention of staining for MLH1, replicating the findings of a recent publication restricted to advanced traditional serrated adenomas, in which no loss of mismatch repair function was observed in 60 cases. ${ }^{15}$ This indicates a crucial biological difference in the pathways by which traditional serrated adenomas and sessile serrated adenomas with dysplasia progress to carcinoma. In the majority of sessile serrated adenomas with dysplasia, MLH1 silencing is a critical step in malignant progression, resulting in microsatellite instability and is associated with an improved prognosis. ${ }^{38}$ In contrast, essentially all colorectal carcinomas arising from traditional serrated adenomas are microsatellite stable.

$\beta$-Catenin is the final transcription factor of the Wnt signaling pathway; activation results in increased transcription of a variety of proliferation promoting genes. ${ }^{39}$ In conventional chromosomal instability-type colorectal carcinoma, the Wnt pathway is activated by $A P C$ mutation. However, $A P C$ mutation is uncommon in serrated pathway carcinomas; instead, a variety of Wnt suppressors can be silenced by promoter methylation allowing activation of Wnt signaling. ${ }^{39}$ A shift from membranous to nuclear staining of $\beta$-catenin is indicative of canonical Wnt pathway activation irrespective of cause and is an effective surrogate for identifying Wnt signaling. Advanced areas of the traditional serrated adenomas showed a highly significant increase in nuclear $\beta$-catenin staining, indicating Wnt pathway activation as an important step in malignant progression. This finding is concordant with other studies of advanced traditional serrated adenomas. ${ }^{14,15}$

TP53 and CDKN2A (encoding p16) are critical tumor suppressor genes with loss of function demonstrated in a wide range of malignancies. Strong nuclear staining for p53 is an effective surrogate for TP53 gene mutation and was seen in the majority of advanced traditional serrated adenomas in this series. ${ }^{40}$ In contrast, increased cytoplasmic or nuclear p16 staining indicates increased production of functional protein. ${ }^{24}$ Normal colonic mucosa is p16 negative, whereas the basal crypts and the ectopic crypt formations of ordinary traditional serrated adenomas show weak and patchy p16 staining; advanced areas frequently show strong p16 expression. This incremental pattern is postulated to represent increasing efforts to control cell proliferation by upregulation of $C D K N 2 A$ expression. However, in the majority of $B R A F$ mutant but not KRAS mutant or BRAF/KRAS wild-type traditional serrated adenomas, p16 expression is abruptly lost in areas of dysplasia 
and/or carcinoma. This loss is attributed to methylation-induced silencing of the CDKN2A gene and appears to be an important step in the development of adenocarcinoma in these polyps. TP53 mutation has been reported previously, both as a feature of BRAF mutant, microsatellite-stable colorectal carcinoma and specifically in advanced traditional serrated adenomas. ${ }^{14,15,33}$ However, to the best of our knowledge, loss of p16 staining has not previously been reported in advanced traditional serrated adenomas. A detailed outline of the proposed molecular pathways by which traditional serrated adenomas progress to carcinoma is provided in Figure 4.

This study has limitations. First, the series is not consecutive, but instead includes cases that we considered classical traditional serrated adenomas. Our inclusion criteria are those we consider to be the most diagnostically specific. Admittedly, this is based on scant evidence and needs to be confirmed in a series specifically addressing diagnostic features. The 2008 paper of Torlakovic et $a l^{13}$ is the best of this kind to date, but was limited to some extent by the nature of the study polyps, in particular, a limited number of tubulovillous adenomas, resulting in a greater emphasis placed on ectopic crypt formations than perhaps is warranted. Second, our series has a definite bias toward larger polyps because ectopic crypt formations were selected as one of our inclusion criteria and because cases were only included if a complete molecular and immunohistochemical analysis could be performed. Generally, this is more achievable in larger polyps. This may have resulted in a greater proportion of advanced traditional serrated adenomas than might be seen in a consecutive series of traditional serrated adenomas.

In our opinion, however, these weaknesses do not detract from the important conclusions of the study. Furthermore, the clinicopathological and molecular data are very similar to that presented in other

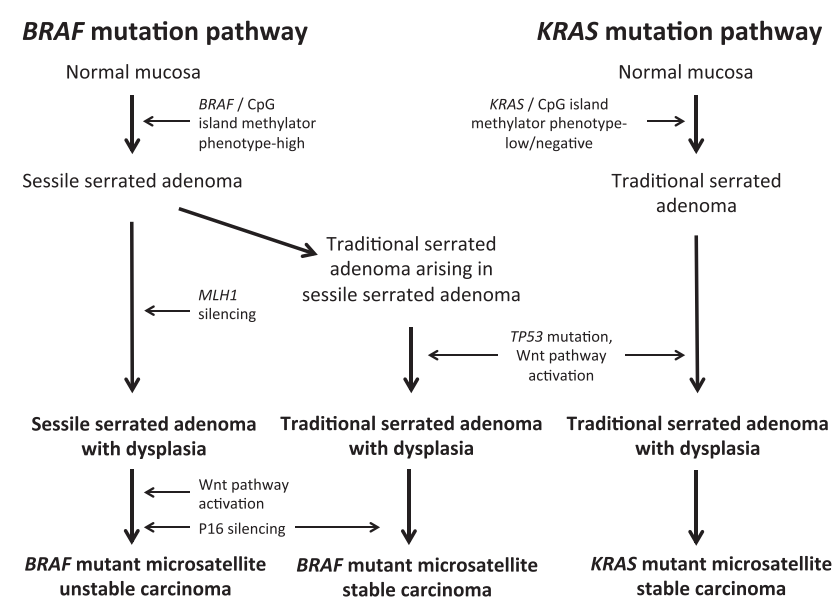

Figure 4 Proposed molecular pathways of malignant progression in $B R A F$ and KRAS mutant traditional serrated adenoma. recent large series. ${ }^{15-17}$ Perhaps most relevant, in a recent study from our group that included 57 consecutive traditional serrated adenomas ( $1 \%$ of all colorectal polyps), the mean size $(11 \mathrm{~mm})$, mean age (62 years), gender distribution ( $50 \%$ female), and anatomical distribution (68\% distal) were similar to what we see in this series. ${ }^{10}$ Advanced histology was not recorded in that study for comparison. Given these similarities, we believe that the data presented here can be inferred to be a reasonable representation of traditional serrated adenomas generally.

In this study, we have built on the current understanding of the morphology and molecular biology of the traditional serrated adenoma. The critical morphological findings are the definite occurrence of traditional serrated adenomas arising in sessile serrated adenomas and microvesicular hyperplastic polyps and the important distinction between this process and development of sessile serrated adenomas with dysplasia. In addition, overt dysplasia arising in a traditional serrated adenoma requires distinction from the senescent change seen in ordinary traditional serrated adenomas. Thus, we believe that the nomenclature of the traditional serrated adenoma should be unified with that of the sessile serrated adenoma, so that the diagnostic categories are traditional serrated adenoma (including traditional serrated adenoma arising in sessile serrated adenoma or microvesicular hyperplastic polyp) and traditional serrated adenoma with dysplasia (to encompass cases with a discrete focus of overt dysplasia). Similar to the sessile serrated adenoma, this approach will simplify the nomenclature and is also more representative of the underlying biology.

At a molecular level, traditional serrated adenomas can be broadly divided into BRAF and KRAS mutant subtypes (with $B R A F / K R A S$ wild-type cases segregating better with KRAS mutant polyps). This distinction has clinicopathological and biological significance. BRAF mutant traditional serrated adenomas are more often proximal, are regularly associated with a precursor polyp, and are more frequently $\mathrm{CpG}$ island methylator phenotype high. In addition, $C D K N 2 A$ silencing appears to be critical to malignant progression of $B R A F$ mutant traditional serrated adenomas but not KRAS or BRAF/KRAS wild-type traditional serrated adenomas.

More broadly, essentially all advanced traditional serrated adenomas show retention of MLH1 staining, implying a near universal microsatellite-stable phenotype. This is expected for KRAS and BRAF/ KRAS wild-type traditional serrated adenomas but was unexpected for BRAF mutant cases. This finding is particularly important given that the $B R A F$ mutant, microsatellite-stable subtype of colorectal carcinoma is known to be highly aggressive. Thus, BRAF mutant traditional serrated adenomas are an important precursor of these aggressive cancers. 
At present, advanced traditional serrated adenomas are not formally recognized. Hence, the surveillance guidelines issued by the US Multi-Society Task Force for Colorectal Carcinoma also do not include this entity. ${ }^{41}$ Given the rarity of these polyps, it is unlikely that high- quality evidence to direct surveillance will become available; however, based on our molecular results, advanced traditional serrated adenomas are potentially aggressive lesions and we believe that in the rare instances when these polyps are identified, complete resection with close surveillance is required.

\section{Acknowledgments}

We gratefully acknowledge NHMRC grant funding (ID: 1063105). Mark Bettington gratefully acknowledges $\mathrm{PhD}$ scholarship funding from Cancer Council Queensland. Thanks also to Madeleine Flynn (Graphics Support Unit, QIMR Berghofer Medical Research Institute) for assistance with image production.

\section{Disclosure/conflict of interest}

The authors declare no conflict of interest.

\section{References}

1 Snover DC. Update on the serrated pathway to colorectal carcinoma. Hum Pathol 2011;42:1-10.

2 Leggett B, Whitehall V. Role of the serrated pathway in colorectal cancer pathogenesis. Gastroenterology 2010;138:2088-2100.

3 Bettington M, Walker N, Clouston A, et al. The serrated pathway to colorectal carcinoma: current concepts and challenges. Histopathology 2013;62:367-386.

4 Jass JR. Classification of colorectal cancer based on correlation of clinical, morphological and molecular features. Histopathology 2007;50:113-130.

5 Toyota M, Ahuja N, Ohe-Toyota M, et al. CpG island methylator phenotype in colorectal cancer. Proc Natl Acad Sci USA 1999;96:8681-8686.

6 Bird AP. CpG-rich islands and the function of DNA methylation. Nature 1986;321:209-213.

7 Longacre TA, Fenoglio-Preiser CM. Mixed hyperplastic adenomatous polyps/serrated adenomas. A distinct form of colorectal neoplasia. Am J Surg Pathol 1990; 14:524-537.

8 Carr NJ, Mahajan H, Tan KL, et al. Serrated and nonserrated polyps of the colorectum: their prevalence in an unselected case series and correlation of BRAF mutation analysis with the diagnosis of sessile serrated adenoma. J Clin Pathol 2009;62:516-518.

9 Spring KJ, Zhao ZZ, Karamatic R, et al. High prevalence of sessile serrated adenomas with BRAF mutations: a prospective study of patients undergoing colonoscopy. Gastroenterology 2006;131:1400-1407.

10 Bettington M, Walker N, Rosty C, et al. Critical appraisal of the diagnosis of the sessile serrated adenoma. Am J Surg Pathol 2014;38:158-166.

11 Bosman FT. World Health OrganizationInternational Agency for Research on Cancer. WHO classification of tumours of the digestive system 2010; Chapter 8:160-166.
12 Torlakovic E, Skovlund E, Snover DC, et al. Morphologic reappraisal of serrated colorectal polyps. Am J Surg Pathol 2003;27:65-81.

13 Torlakovic EE, Gomez JD, Driman DK, et al. Sessile serrated adenoma (SSA) vs traditional serrated adenoma (TSA). Am J Surg Pathol 2008;32:21-29.

14 Fu B, Yachida S, Morgan R, et al. Clinicopathologic and genetic characterization of traditional serrated adenomas of the colon. Am J Clin Pathol 2012;138:356-366.

15 Tsai JH, Liau JY, Lin YL, et al. Traditional serrated adenoma has two pathways of neoplastic progression that are distinct from the sessile serrated pathway of colorectal carcinogenesis. Mod Pathol; advance online publication, 7 March 2014; doi:10.1038/modpathol. 2014.35 (e-pub ahead of print).

16 Kim MJ, Lee EJ, Suh JP, et al. Traditional serrated adenoma of the colorectum: clinicopathologic implications and endoscopic findings of the precursor lesions. Am J Clin Pathol 2013;140:898-911.

17 Kim KM, Lee EJ, Kim YH, et al. KRAS mutations in traditional serrated adenomas from Korea herald an aggressive phenotype. Am J Surg Pathol 2010;34: 667-675.

18 Yantiss RK, Oh KY, Chen YT, et al. Filiform serrated adenomas: a clinicopathologic and immunophenotypic study of 18 cases. Am J Surg Pathol 2007;31: 1238-1245.

19 Ha SY, Lee SM, Lee EJ, et al. Filiform serrated adenoma is an unusual, less aggressive variant of traditional serrated adenoma. Pathology 2012;44:18-23.

20 Kim YH, Kakar S, Cun L, et al. Distinct CpG island methylation profiles and BRAF mutation status in serrated and adenomatous colorectal polyps. Int J Cancer 2008;123:2587-2593.

21 Rex DK, Ahnen DJ, Baron JA, et al. Serrated lesions of the colorectum: review and recommendations from an expert panel. Am J Gastroenterol 2012;107:1315-1329; quiz 1314, 1330.

22 Makinen MJ. Colorectal serrated adenocarcinoma. Histopathology 2007;50:131-150.

23 Yachida S, Mudali S, Martin SA, et al. Beta-catenin nuclear labeling is a common feature of sessile serrated adenomas and correlates with early neoplastic progression after BRAF activation. Am J Surg Pathol 2009; 33:1823-1832.

24 Kriegl L, Neumann J, Vieth M, et al. Up and downregulation of p16(Ink4a) expression in BRAF-mutated polyps/adenomas indicates a senescence barrier in the serrated route to colon cancer. Mod Pathol 2011;24: 1015-1022.

25 Young J, Barker MA, Simms LA, et al. Evidence for BRAF mutation and variable levels of microsatellite instability in a syndrome of familial colorectal cancer. Clin Gastroenterol Hepatol 2005;3:254-263.

26 Whitehall V, Tran K, Umapathy A, et al. A multicenter blinded study to evaluate KRAS mutation testing methodologies in the clinical setting. J Mol Diagn 2009;11:543-552.

27 Weisenberger DJ, Siegmund KD, Campan M, et al. CpG island methylator phenotype underlies sporadic microsatellite instability and is tightly associated with BRAF mutation in colorectal cancer. Nat Genet 2006; 38:787-793.

28 Burnett-Hartman AN, Newcomb PA, Potter JD, et al. Genomic aberrations occurring in subsets of serrated colorectal lesions but not conventional adenomas. Cancer Res 2013;73:2863-2872. 
29 Park SJ, Rashid A, Lee JH, et al. Frequent CpG island methylation in serrated adenomas of the colorectum. Am J Pathol 2003;162:815-822.

30 Hafezi-Bakhtiari S, Serra S, Colling R, et al. Tubulovillous/villous adenomas and traditional serrated adenomas in the left colon and rectum show histological overlap. Mod Pathol 2014;27:161-212; (abstract 722).

31 Kim MJ, Lee EJ, Chun SM, et al. Pedunculated serrated polyp with histologic features of sessile serrated adenoma: a clinicopathologic and molecular study. Am J Surg Pathol 2013;37:1039-1043.

32 Goldstein NS. Small colonic microsatellite unstable adenocarcinomas and high-grade epithelial dysplasias in sessile serrated adenoma polypectomy specimens: a study of eight cases. Am J Clin Pathol 2006;125:132-145.

33 Bond CE, Umapathy A, Ramsnes I, et al. p53 mutation is common in microsatellite stable, BRAF mutant colorectal cancers. Int J Cancer 2012;130:1567-1576.

34 Makinen MJ, George SM, Jernvall P, et al. Colorectal carcinoma associated with serrated adenoma-prevalence, histological features, and prognosis. J Pathol 2001;193:286-294.

35 Stefanius K, Ylitalo L, Tuomisto A, et al. Frequent mutations of KRAS in addition to BRAF in colorectal serrated adenocarcinoma. Histopathology 2011;58:679-692.
36 O’Brien MJ, Yang S, Mack C, et al. Comparison of microsatellite instability, CpG island methylation phenotype, BRAF and KRAS status in serrated polyps and traditional adenomas indicates separate pathways to distinct colorectal carcinoma end points. Am J Surg Pathol 2006;30:1491-1501.

37 Gurzu S, Jung I. Aberrant pattern of the cytokeratin 7/cytokeratin 20 immunophenotype in colorectal adenocarcinomas with BRAF mutations. Pathol Res Pract 2012;208:163-166.

38 Ribic CM, Sargent DJ, Moore MJ, et al. Tumor microsatellite-instability status as a predictor of benefit from fluorouracil-based adjuvant chemotherapy for colon cancer. N Engl J Med 2003;349:247-257.

39 MacDonald BT, Tamai K, He X. Wnt/beta-catenin signaling: components, mechanisms, and diseases. Dev Cell 2009;17:9-26.

40 Baas IO, Mulder JW, Offerhaus GJ, et al. An evaluation of six antibodies for immunohistochemistry of mutant p53 gene product in archival colorectal neoplasms. J Pathol 1994;172:5-12.

41 Lieberman DA, Rex DK, Winawer SJ, et al. Guidelines for colonoscopy surveillance after screening and polypectomy: a consensus update by the US MultiSociety Task Force on Colorectal Cancer. Gastroenterology 2012;143:844-857. 